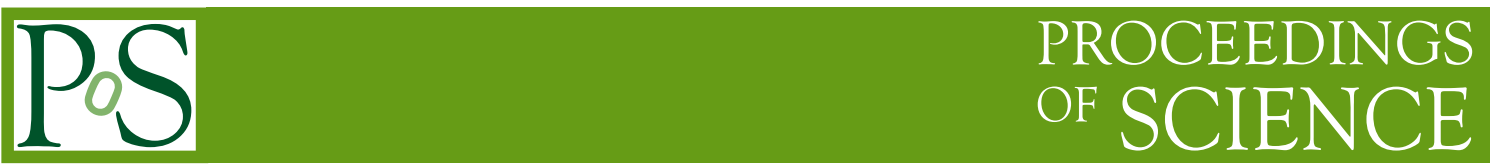

\title{
Mini-EUSO optics design and tests
}

\section{Yoshiyuki TAKIZAWA*}

RIKEN, Japan

E-mail: takky@riken.jp

\section{Hiroshi Kasuga}

RIKEN, Japan

\section{Hitoshi Ohmori}

RIKEN, Japan

\section{Marco Casolino}

RIKEN, Japan

\section{Toshikazu Ebisuzaki}

RIKEN, Japan

\section{for the JEM-EUSO Collaboration}

Mini-EUSO ("UV atmosphere" in Russian Space Program) is a science mission to observe UV phenomena in the night Earth atmosphere from a UV transparent porthole of the International Space Station and will serve as a pathfinder for future ultra-high energy cosmic ray missions such as KLYPVE-EUSO (K-EUSO) and JEM-EUSO. The Mini-EUSO optical design has two $250 \mathrm{~mm}$ double-sided Fresnel lenses and a flat focal surface. The overall length of the optical system is 300 $\mathrm{mm}$. Its field of view is about $\pm 22^{\circ}$. The RMS spot sizes between $0^{\circ}$ and $22^{\circ}$ field angle are 2.0 $\mathrm{mm} \sim 3.5 \mathrm{~mm}$ in diameter, and conform to the pixel size of the M64 multi-anode photomultiplier (HAMAMATSU Photonics). The material of lenses is UV transparent PMMA (Poly Methyl Methacrylate, PMMA-000, MITSUBISHI RAYON) and the lens thickness is $11 \mathrm{~mm}$. The lenses were manufactured using the diamond turning machine by the Materials Fabrication Laboratory of RIKEN. We confirmed that the surface roughness of lenses is less than $20 \mathrm{~nm}$ (RMS). In this paper, we will describe the details of the optical system and test results.

35th International Cosmic Ray Conference - ICRC2017

10-20 July, 2017

Bexco, Busan, Korea

\footnotetext{
* Speaker.
} 


\section{Mini-EUSO optical design}

Mini-EUSO ("UV atmosphere" in Russian Space Program) is a science mission to observe UV phenomena in the night Earth atmosphere from a UV transparent porthole of the International Space Station and will serve as a pathfinder for future ultra-high energy cosmic ray missions such as KLYPVE-EUSO (K-EUSO) and JEM-EUSO[四[[]]. The optical system design for the MiniEUSO mission consists of two double sided flat Fresnel lenses and a flat focal surface. In the original design in 2015[䧃], the distance between the font lens and the focal surface, which we call overall length, was $366 \mathrm{~mm}$. After the request of the system design, the optics was re-designed to reduce the overall length to $300 \mathrm{~mm}$. Field Of View (FOV) was $\pm 19^{\circ}$ and curved focal surface was

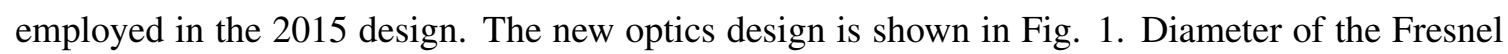
lens is $250 \mathrm{~mm}$. The Mini-EUSO optics has a low focal number F\# 0.6, and the effective focal length is $150 \mathrm{~mm}$. Its FOV is wider than $\pm 19^{\circ}$ of the older design. The details of FOV is described in next section. The material of Fresnel lens is UV transparent PMMA (Poly Methyl Methacrylate, PMMA-000, MITSUBISHI RAYON)[可]. The thickness and the mass of each lens are $11 \mathrm{~mm}$ and $0.9 \mathrm{~kg}$, respectively. The characteristics of Fresnel lenses are shown in Table $\mathrm{W}$.

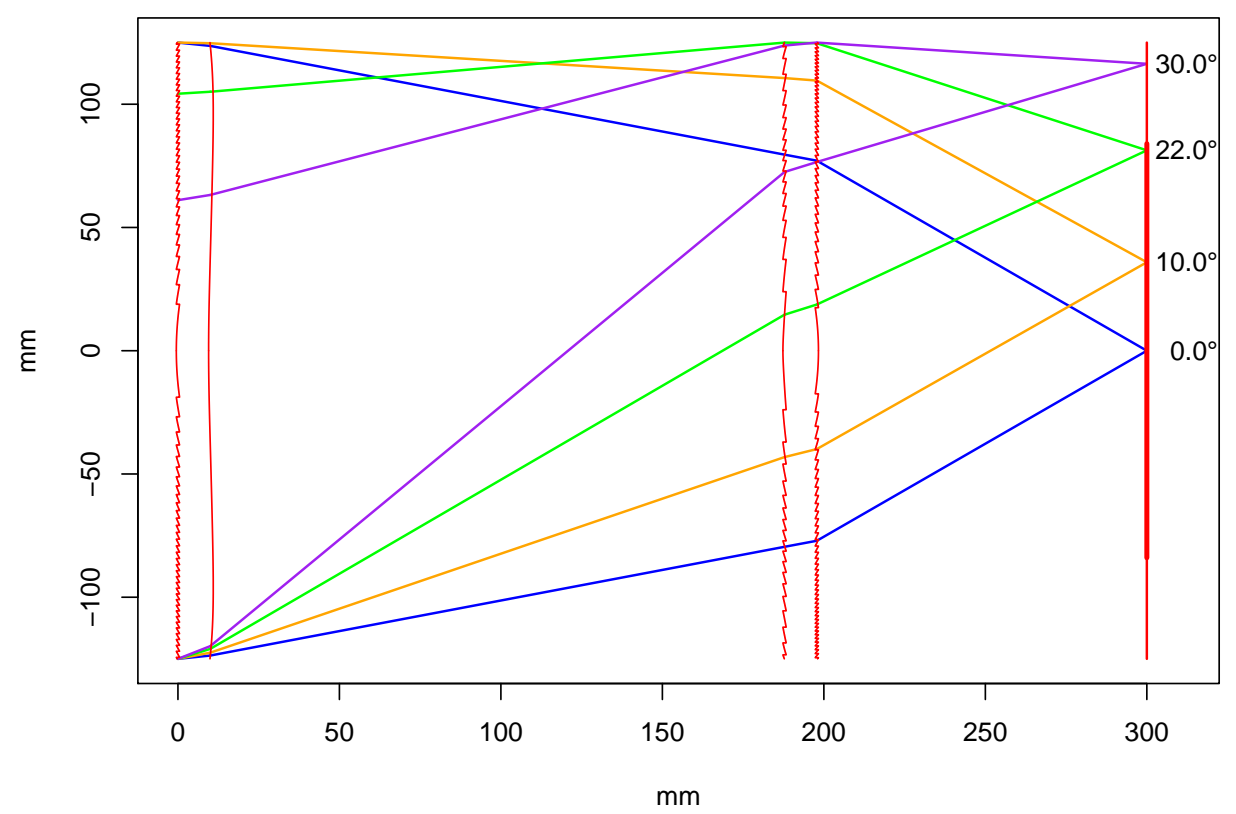

Figure 1: The optical design of the Mini-EUSO telescope.

\section{Field of view and expected performance}

The Photo Detector Module (PDM, Mini-EUSO focal surface detector) is an $6 \times 6$ array of M64 multi-anode photomultipliers (HAMAMATSU Photonics)[3]. Field of View (FOV) for the opposite sides distance of PDM is $\pm 22^{\circ}$. FOV for the diagonal distance of PDM is $\pm 30^{\circ}$, because PDM detection area is square plane. FOV of PDM is shown in Fig. $\square$. 
Table 1: Characteristics of lens surfaces. Groove height is $1 \mathrm{~mm}$.

\begin{tabular}{|l|c|c|c|c|}
\hline Lens & Surface & Surface type & Groove pitch $(\mathrm{mm})$ & Number of grooves \\
\hline \hline \multirow{3}{*}{ Front lens } & Front & Fresnel & $2.29 \sim 18.85$ & 35 \\
\cline { 2 - 5 } & Back & Aspherical & N/A & N/A \\
\hline \multirow{3}{*}{ Rear lens } & Front & Fresnel & $4.19 \sim 23.94$ & 18 \\
\cline { 2 - 5 } & Back & Fresnel & $1.50 \sim 17.47$ & 42 \\
\hline
\end{tabular}

FOV vs Radius position on FS
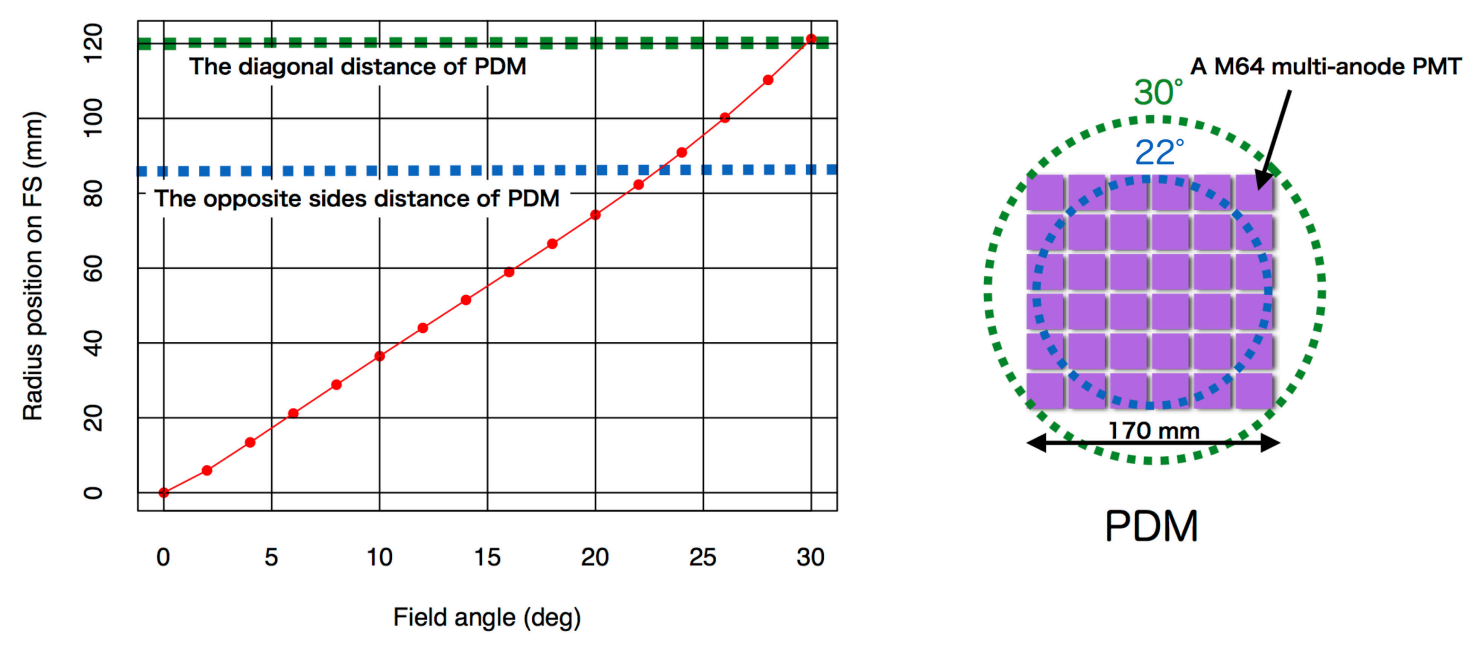

Figure 2: Field of view of the photo detector module. Left plot shows the focusing positions on the PDM as function of field angle. Right schematic figure shows the maximum FOV on the PDM. The blue dash line is FOV of $22^{\circ}$ for the opposite sides distance of PDM and the green dash line is FOV of $30^{\circ}$ for the diagonal distance of PDM.

The point spread function of the optical system using raytracing simulation is shown in Fig. [3. The Photon Collection Efficiencies (PCE) in a $3.3 \mathrm{~mm}$ diameter is $\sim 45 \%$ which takes into account of loss factors ( surface reflection, material absorption, surface roughness, Fresnel facet back cut, manufacturing loss). The $3.3 \mathrm{~mm}$ diameter circle is equivalent area of a pixel ( $2.88 \mathrm{x}$ $2.88 \mathrm{~mm}^{2}$ ). The manufacturing loss is caused by a tip shape of a tool bite[ 6$]$. Since the bit tip has a hemispherical shape with a $0.05 \mathrm{~mm}$ radius the bottom of the Fresnel facet back cut has this radius and does not contribute to the lens performance. PCE is calculated with a formula with our dedicated raytrace simulation (D.

$$
P C E=\frac{\text { Photon counts within a circle of } 3.3 \mathrm{~mm} \text { diameter on the focal surface }}{\text { Photon counts which have passed the front lens }}
$$



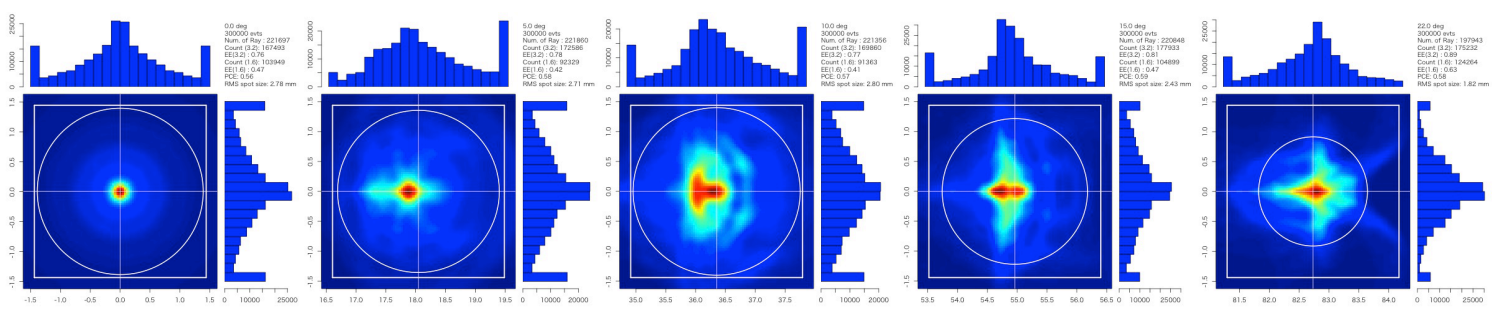

Figure 3: Point spread function of the optical system for in-comming parallel light rays at different inclination angles (from left to right, $0^{\circ}, 5^{\circ}, 10^{\circ}, 15^{\circ}$ and $22^{\circ}$ ). The white square is a size of pixel of MAPMT and the white circle is RMS spot size.

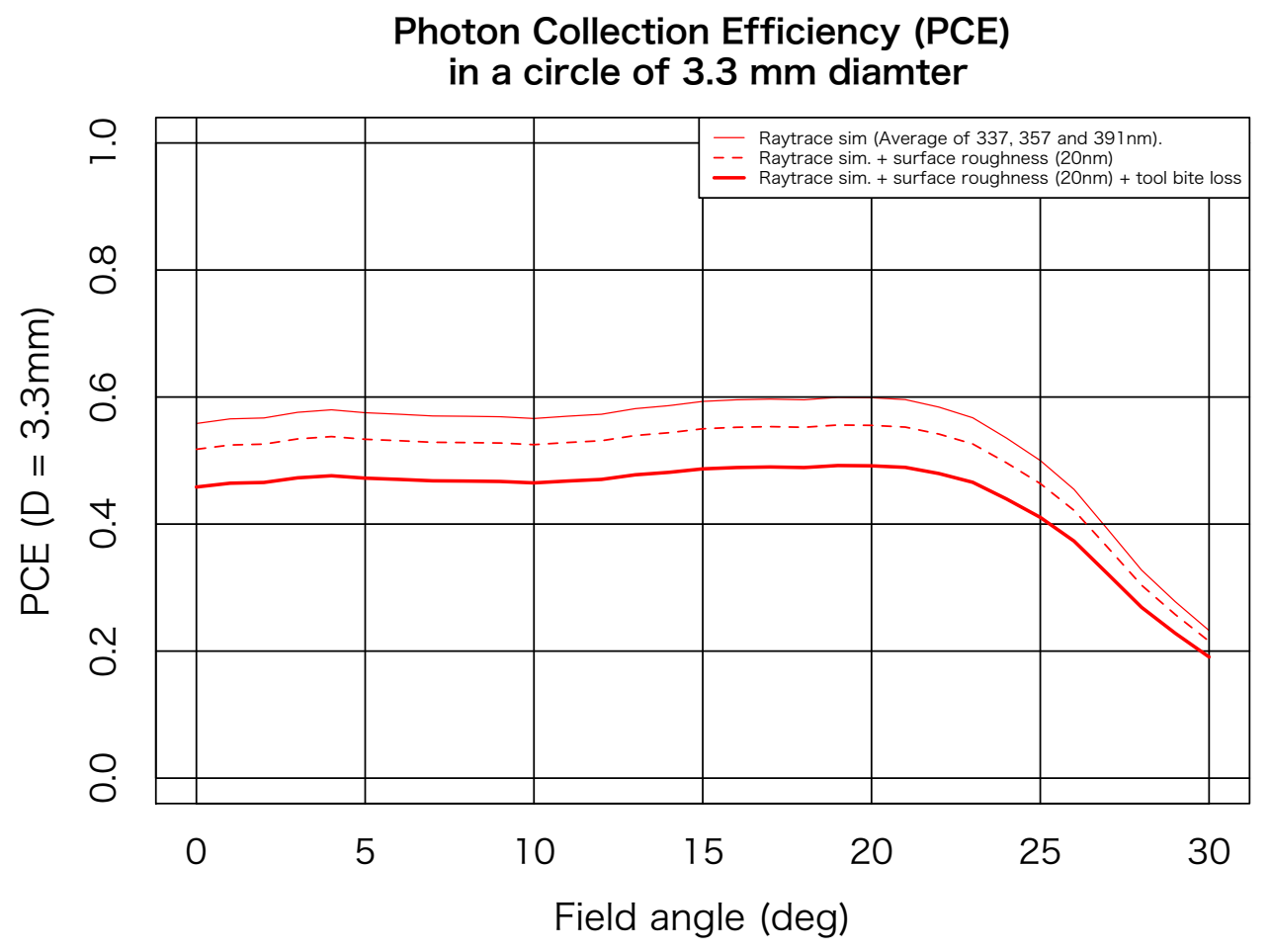

Figure 4: Photon collection efficiencies (PCE) of the optical system for light coming from showers of different inclination between $0^{\circ}$ and $30^{\circ}$ ). The bold red curve indicates an expected efficiency which takes into account of surface roughness of $20 \mathrm{~nm}$ and tool bite loss.

\section{Lens manufacturing}

Lenses were manufactured using the UPL-240M (RIKEN SEIKO CO., LTD.) machine. The UPL-240M has a turn table for rotating a work material and three movable axises with $1.4 \mathrm{~nm}$ resolution for controlling a tool bite position. According to the requirement, the surface roughness of lenses should be smaller than $20 \mathrm{~nm}$ RMS. This is the most important requirement to meet the observation performance. The surface roughness has a direct impact on to overall optics transmission. We have developed a technology to obtain smooth surfaces through lenses manufacturing efforts for previous JEM-EUSO submissions (Bread Board Model of JEM-EUSO, EUSO-TA, EUSO- 
Balloon, EUSO-SPB) $[$ [ $][$ [ $]$. We used an optical surface profiler using interferometer system measurements as evaluation the requirement of surface roughness. The results of surface measurements are shown in Table $\rrbracket$. All measured values are smaller than $20 \mathrm{~nm}$ as the requirement.

Table 2: Measured RMS surface roughness of lenses using the optical surface profiler.

\begin{tabular}{|l|c|c|c|c|}
\hline Lens & Surface & Central area & Middle area & Outer area \\
\hline \hline \multirow{3}{*}{ Front lens } & Front & $16.6 \mathrm{~nm}$ & $18.2 \mathrm{~nm}$ & $14.1 \mathrm{~nm}$ \\
\cline { 2 - 5 } & Back & $16.7 \mathrm{~nm}$ & $13.2 \mathrm{~nm}$ & $15.6 \mathrm{~nm}$ \\
\hline \multirow{3}{*}{ Rear lens } & Front & $19.1 \mathrm{~nm}$ & $18.2 \mathrm{~nm}$ & $16.9 \mathrm{~nm}$ \\
\cline { 2 - 5 } & Back & $16.9 \mathrm{~nm}$ & $16.5 \mathrm{~nm}$ & $16.0 \mathrm{~nm}$ \\
\hline
\end{tabular}

\section{Optics tests}

\subsection{Measurement system for lens tests}

We built a lens measurement system shown in Fig. [1] to evaluate the performance such as angle of refraction, transmittance, and spot diagram. It consists of light sources, CCD detector (detection area: $6.45 \mathrm{~mm} \times 4.84 \mathrm{~mm}$ ) and five motorized linear slides. The light sources are set up on an motorized linear slide and CCD are set up on the other linear slide. There are two types of light sources, near-UV laser (405nm) and the point light source using LED (355, 375, $393 \mathrm{~nm})$. The near-UV laser was used to test single lens to measure refraction angle by position by position on the lens and the point source tests the optical system to measure optical throughput (PCE) and spot image. CCD has a small motorized linear slide to scan focusing position. The front lens and rear lens are located on separate linear slides to move independently between the light sources and CCD.

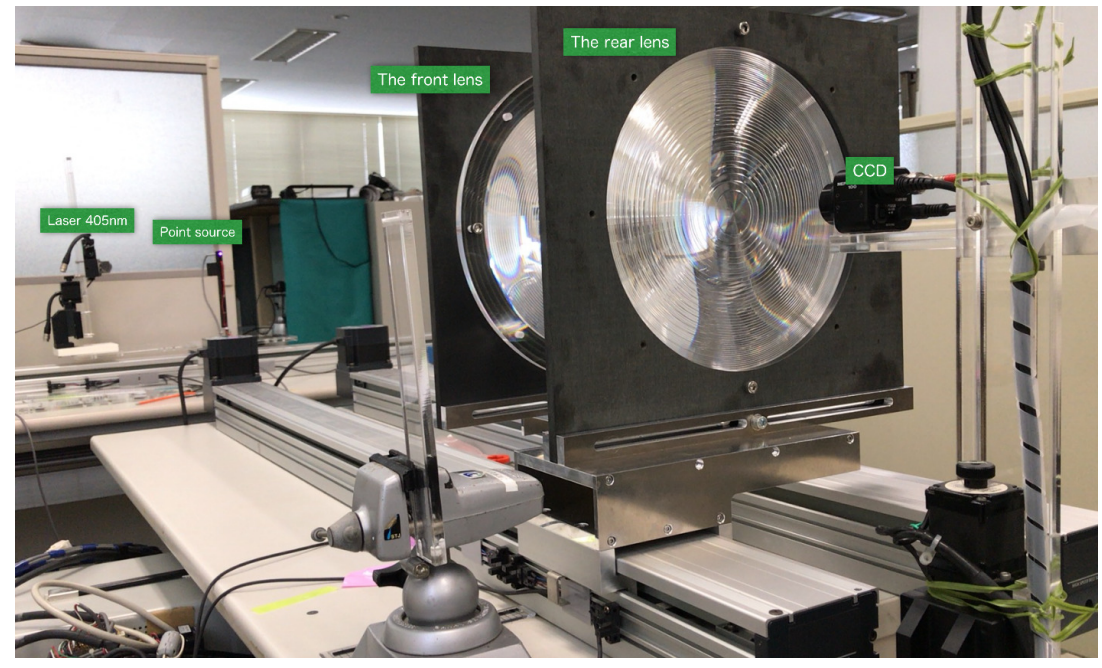

Figure 5: The measurement system for optics performance. 


\subsection{The single lens test (the front lens and the rear lens)}

The front lens and the rear lens are measured by near-UV laser $(405 \mathrm{~nm})$. The laser illuminates perpendicularly on the front surface of the lens and is scanned along the radial direction to measure in-situ refracted power. Measured refraction angle of the front lens and rear lens is plotted in Fig.6. In comparison with the expected curves (red solid lines) of a mathematical design model, they agree well with each other within error about $\pm 1^{\circ}$, which corresponds with the laser beam size (2 $\mathrm{mm}$ in diameter). Two measured points of real lens at $15 \mathrm{~mm}$ and $125 \mathrm{~mm}$ radius are not on the expected curve due to pass the fresnel edge.

The single lens test

(Front lens and Rear lens)

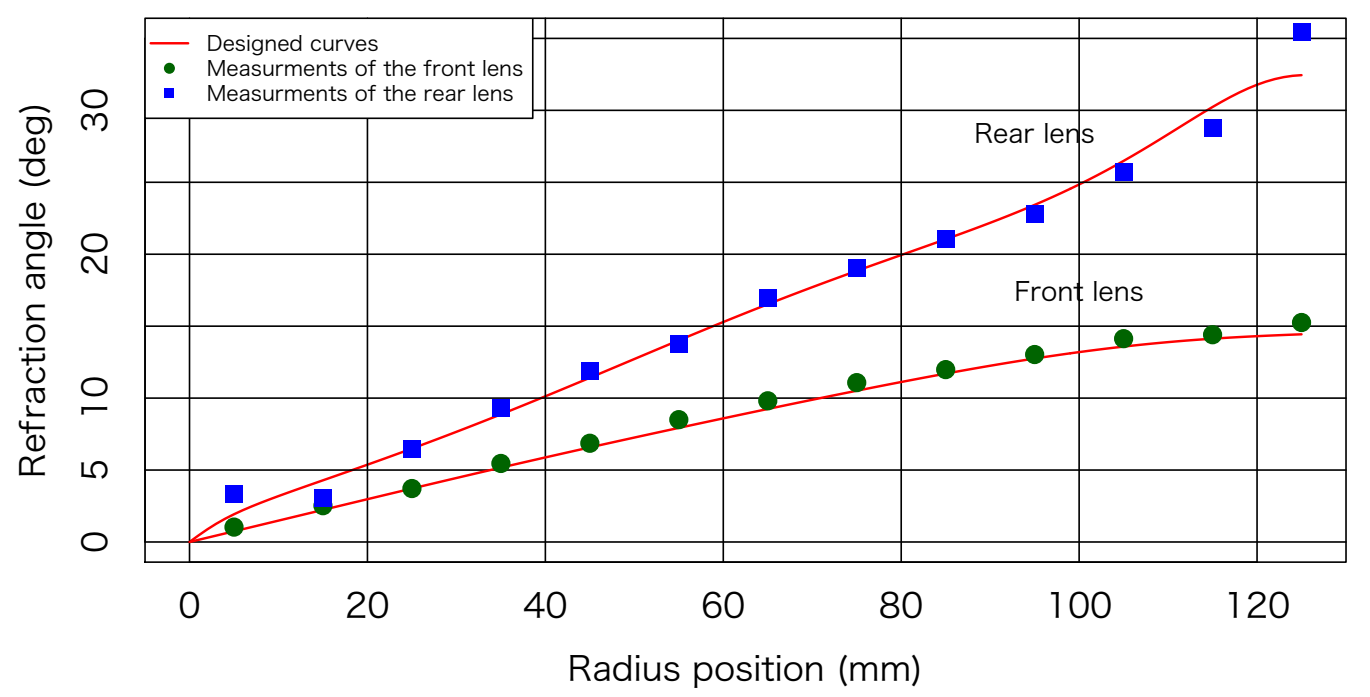

Figure 6: The refraction angle of the front lens and the rear lens using the $405 \mathrm{~nm}$ UV laser. The red solid curves are expected refraction angle as function of radius of lens. The square and dot show measured results.

\subsection{The Mini-EUSO optics tests using point sources}

The Mini-EUSO optics were tested using point sources (LED: 355, 375, $393 \mathrm{~nm}$ ) to measure optics throughput (PCE) and point spread function (PSF). The distance from the point source to the front lens is $1660 \mathrm{~mm}$. The expected focusing position using this point source shifts $25.6 \mathrm{~mm}$ in a direction away from the design position of the focal surface for parallel light rays. Incident light intensity of the point sources are measured with a CCD at $32 \mathrm{~mm}$. This distance is estimated form a geometrical similar area of the point source irradiating the front lens area. The expected PSFs are simulated with same configuration and the light emission pattern. Spot images of the point source ( $355 \mathrm{~nm}$ LED) are shown in Fig.D. Observed spot images have correlation well with simulated PSF plots. First results of the PCE measurement are shown in Table 4.3. Their measured values are as expected from the raytrace simulation including surface roughness loss (20 nm RMS) and tool bite loss. We, therefor, estimate that actual PCE is $\sim 45 \%$ from the raytracing simulation for parallel rays. The accuracy of the present PCE is estimated as better than $10 \%$ and we are improving the 
measurement.
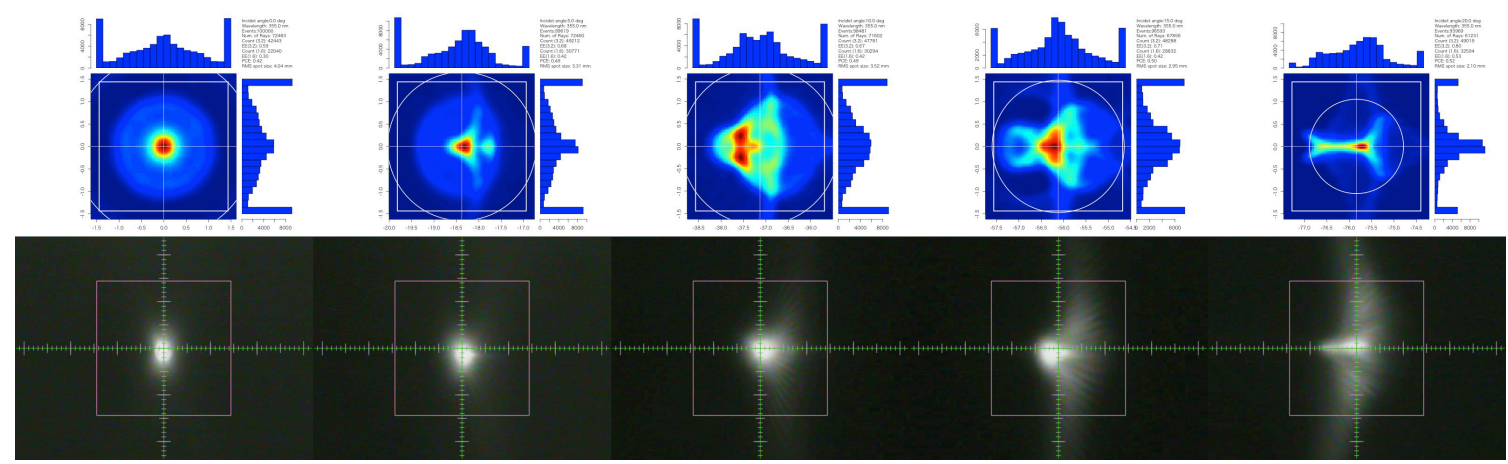

Figure 7: Spot diagrams of the raytrace simulation (up) and measured spot images (bottom) for the point source (355 nm LED at $1.66 \mathrm{~m}$ distance) with different inclination (from left to right, $0^{\circ}, 5^{\circ}, 10^{\circ}, 15^{\circ}$ and $20^{\circ}$ ). The square, in plots and images, is a size of pixel of MAPMT.

Table 3: The PCE measurement results of the Mini-EUSO optics using LED point sources (355, 375, 393 $\mathrm{nm})$

\begin{tabular}{|l|c|c|c|c|c|c|}
\hline Wavelength & Data type & FOV $^{\circ}$ & FOV $^{\circ}$ & ${\text { FOV } 10^{\circ}}^{\text {FOV } 15^{\circ}}$ & FOV 20 $^{\circ}$ \\
\hline \hline \multirow{3}{*}{$355 \mathrm{~nm}$} & Measurement & 0.27 & 0.32 & 0.44 & 0.48 & 0.42 \\
\cline { 2 - 7 } & Expected & 0.34 & 0.39 & 0.39 & 0.40 & 0.43 \\
\hline \multirow{3}{*}{$375 \mathrm{~nm}$} & Measurement & 0.35 & 0.44 & 0.47 & 0.45 & 0.41 \\
\cline { 2 - 7 } & Expected & 0.40 & 0.45 & 0.45 & 0.46 & 0.47 \\
\hline \multirow{3}{*}{$393 \mathrm{~nm}$} & Measurement & 0.62 & 0.47 & 0.45 & 0.42 & 0.44 \\
\cline { 2 - 7 } & Expected & 0.55 & 0.50 & 0.48 & 0.48 & 0.49 \\
\hline
\end{tabular}

\section{Conclusion}

We have manufactured the Mini-EUSO optics and its optical performances have satisfied the requirements. The surface roughness is lower than the $20 \mathrm{~nm}$ RMS of the requirement and the measured spot images using the LED source $(355 \mathrm{~nm})$ have correlated well with the raytrace simulation. The optics throughputs (PCE) using LED point source are consistent with the raytracing simulation. We estimate that actual PCE is $\sim 45 \%$ from the raytracing simulation for parallel rays. We are planning tests using parallel beam (300 $\mathrm{mm}$ in diameter) to measure more precise optical throughputs with different inclination than the point source measurements. 


\section{Acknowledgments:}

We dedicate this work to Yoshiya Kawasaki and Jacek Karczmarczyk, members of the JEM- EUSO Collaboration, who ultimately passed away in 2016.

This work was partially supported by NASA award 11-APRA-0058 in the USA, by Basic Science Interdisciplinary Research Projects of RIKEN and JSPS KAKENHI Grant (22340063, 23340081, and 24244042), by the Italian Ministry of Foreign Affairs, General Direction for the Cultural Promotion and Cooperation, by the Italian Space Agency ASI contract 2016-1-U.0 "Mini-EUSO, by the ' Helmholtz Alliance for Astroparticle Physics HAP' funded by the Initiative and Networking Fund of the Helmholtz Association, Germany, and by Slovak Academy of Sciences MVTS JEMEUSO as well as VEGA grant agency project 2/0076/13. Russia is supported by ROSCOSMOS and the Russian Foundation for Basic Research Grant No 13-02-12175-ofi-m, by Grant No 15-35-21038-mol-a-ved and No 16-29-13065. The Spanish Consortium involved in the JEM-EUSO Space Mission is funded by MICINN \& MINECO under the Space Program projects: AYA2009- 06037-E/AYA, AYA-ESP2010-19082, AYA-ESP2011-29489-C03, AYA-ESP2012-39115-C03, AYA-ESP2013-47816- C4, MINECO/FEDER-UNAH13-4E-2741, CSD2009-00064 (Consolider MULTIDARK) and by Comunidad de Madrid (CAM) under projects S2009/ESP-1496 \& S2013/ICE-2822.

\section{References}

[1] J.H. Adams Jr. et al. - JEM-EUSO Collaboration, "An evaluation of the exposure in nadir observation of the JEM-EUSO mission", Astrop. Phys. 44 (2013) 76

[2] P. Klimov et al. - JEM-EUSO Collaboration, "Status of the KLYPVE-EUSO detector for UHECR study on board the ISS", Proc. 35th ICRC, 2017

[3] M. Ricci et al. - JEM-EUSO Collaboration "Mini-EUSO: a precursor mission to observe Atmosphere and Earth UV emission from the International Space Station", Proc. 35th ICRC, 2017

[4] M. Ricci et al. - JEM-EUSO Collaboration "Mini-EUSO: a pathfinder for JEM-EUSO to measure Earth's UV background from the ISS", 34th ICRC, 2015

[5] Y. Takizawa et al. - JEM-EUSO Collaboration "The TA-EUSO and EUSO-Balloon optics designs", 33rd ICRC, 2013

[6] Y. Hachisu et al. - JEM-EUSO Collaboration "Manufacturing of the TA-EUSO and EUSO-Balloon lenses", 33rd ICRC, 2013

[7] Y. Hachisu et al. - JEM-EUSO Collaboration "JEM-EUSO lens manufacturing", 32nd ICRC, 2011 